\title{
Psychometric properties of the Anxiety Inventory for Respiratory Disease in patients with COPD in China
}

Xiao-Yan Dong, ${ }^{1, *}$ Lan Wang, ',* Yan-Xia Tao, ' Xiu-li Suo, ${ }^{2}$ Yue-Chuan Li, ${ }^{2}$ Fang Liu,' 'Yue Zhao,' Qing Zhang'

'School of Nursing, Tianjin Medical University, ${ }^{2}$ Department of Respiratory Care, Tianjin Chest Hospital, Tianjin, People's Republic of China

*These authors contributed equally to this work

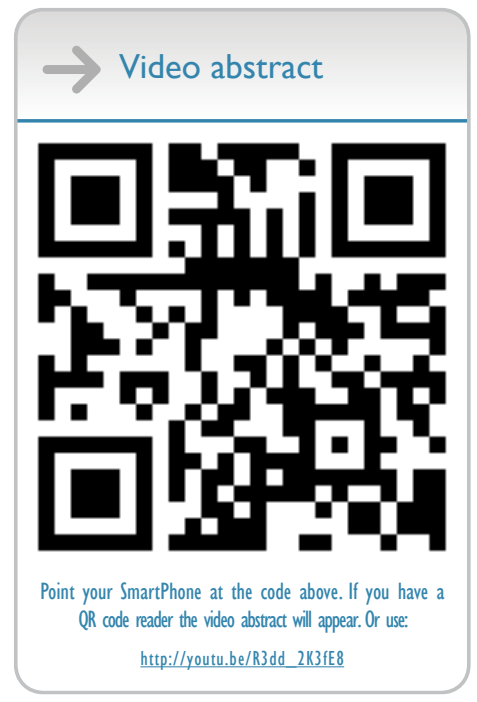

Correspondence: Yue Zhao; Qing Zhang School of Nursing, Tianjin Medical University, 22 Qi Xiang Tai Road, Heping District, Tianjin 300070, People's Republic of China Tel +86228333 690I; +862283336907 Fax +862283336900 Email yuezhao35@hotmail.com; snzhangqing@126.com
This article was published in the following Dove Press journal: International Journal of COPD

20 December 2016

Number of times this article has been viewed

Background: Anxiety is a common comorbidity in patients with COPD in China, and it can significantly decrease patients' quality of life. Almost all anxiety measurements contain somatic items that can overlap with symptoms of COPD and side effects of medicines, which can lead to bias in measuring anxiety in patients with COPD. Therefore, a brief and diseasespecific non-somatic anxiety measurement scale, the Anxiety Inventory for Respiratory Disease (AIR), which has been developed and validated in its English version, is needed for patients with COPD in China.

Methods: A two-center study was conducted in two tertiary hospitals in Tianjin, China. A total of 181 outpatients with COPD (mean age $67.21 \pm 8.10$ years, $32.6 \%$ women), who met the inclusion and exclusion criteria, were enrolled in the study. Test-retest reliability was examined using intraclass correlation coefficients. The internal consistency was calculated by Cronbach's $\alpha$. Content validity was examined using the Content Validity Index (CVI), scale-level CVI/universal agreement, and scale-level CVI/average agreement (S-CVI/Ave). Besides, convergent validity and construct validity were also examined.

Results: The AIR-C (AIR-Chinese version) scale had high test-retest reliability (intraclass correlation coefficient $=0.904$ ) and internal consistency (Cronbach's $\alpha=0.914$ ); the content validity of the AIR-C scale was calculated by CVI, scale-level CVI/universal agreement, and S-CVI/Ave at values of 0.89-1, 0.90, and 0.98, respectively. Meanwhile, the AIR-C scale had good convergent validity, correlating with the Hospital Anxiety and Depression Scale-Anxiety ( $r=0.81, P<0.01$ ), and there were significant correlations between the AIR-C and Clinical COPD Questionnaire (CCQ; $r=0.44, P<0.01$ ) and Activities of Daily Living Scale (ADLS; $r=0.36$, $P<0.01)$. A two-factor model of general anxiety and panic symptoms in the AIR-C scale had the best fit according to Confirmatory Factor Analysis (CFA).

Conclusion: The AIR-C scale had a good reliability and validity for patients with COPD and can be used as a user-friendly and valid tool for measuring anxiety symptoms among patients with COPD in China.

Keywords: COPD, Anxiety Inventory for Respiratory Disease, reliability, validity

\section{Introduction}

COPD is a major public health issue causing significant disability and mortality worldwide. ${ }^{1}$ The World Health Organization (WHO) predicts that COPD prevalence will continue to increase, becoming the world's third major cause of death by $2030 .^{2}$ In China, a cross-sectional survey of COPD conducted between 2002 and 2004 indicated that the overall prevalence of the disease in people $>40$ years old was $8.2 \% .^{3}$ In addition, COPD is a complicated disease that has multiple comorbidities, which can 
contribute to significant health, social and economic costs for the individual and community and health services. ${ }^{1}$

Of such comorbidities, anxiety is a common psychological comorbidity that is related to worse health outcomes, including higher rates of exacerbations, hospitalization, length of stay, and survival rates after emergency treatment. ${ }^{4}$ Besides, some previous studies indicated that anxiety had a negative effect on health-related quality of life and self-reported functional limitations. ${ }^{5,6}$ The prevalence of anxiety has been found to be significantly higher in patients with COPD than in the general population. ${ }^{7}$ Previous reviews indicated that up to $55 \%$ of patients with COPD may have a clinical anxiety disorder. ${ }^{8,9}$ One cross-sectional study in China showed the prevalence of anxiety at approximately up to $57.1 \% .^{10}$ Despite the high prevalence of anxiety in patients with COPD, it is often neglected and undermanaged. ${ }^{11}$ Besides, methodological weakness and the use of a wide range of diagnostic tools make it difficult to reach a consensus on rates of prevalence. ${ }^{12}$ Previous research indicated that more than two thirds of patients with COPD had not been diagnosed with anxiety. ${ }^{13}$ In China, the specialist clinicians lack the awareness of the knowledge of the mental illness in patients with COPD, and the clinic has no standard measurement tools for measuring anxiety in patients with COPD, which may lead to unmeasured, undiagnosed anxiety in patients with COPD. ${ }^{14,15}$ However, a range of adverse consequences caused by comorbid anxiety cannot be ignored. Therefore, routine measurement of anxiety using well-established measurement tools is essential for patients with COPD in China.

Several self-reported assessment tools are available to measure anxiety in patients with COPD. The Global Initiative for Chronic Obstructive Lung Disease (GOLD) suggests the routine use of self-reported scales such as the Hospital Anxiety and Depression Scale (HADS) and the Beck Anxiety Inventory (BAI) for measuring anxiety. ${ }^{1}$ In China, five instruments have been often used to measure anxiety in patients with COPD, including the HADS, the BAI, the Anxiety Self-report Scales (ASS), the State-Trait Anxiety Inventory (STAI), and the Hamilton Anxiety Scale (HAMA). Nevertheless, several previous studies found that some somatic symptoms of anxiety might overlap with the physical symptoms of patients with COPD and the side effects of medication (eg, heart palpitations, dry mouth, and breathlessness), and they might decrease the validity of these scales. ${ }^{16,17}$

The Anxiety Inventory for Respiratory Disease (AIR) scale, which is a brief, non-somatic, and disease-specific selfreported anxiety tool for measuring anxiety in patients with
COPD, was developed by Professor Yohannes and his team in 2013. ${ }^{18,19}$ The AIR scale is sensitive to change following pulmonary rehabilitation (PR) in patients with COPD and can be used in future studies evaluating interventions that reduce anxiety in patients with COPD. ${ }^{20}$ Besides, it showed excellent internal consistency, test-retest reliability, construct validity, and convergent validity and also had significant correlations with HADS, COPD Assessment Test (CAT), and Manchester Respiratory Activities of Daily Living (MRADL). ${ }^{18}$ The aims of this study included three stages: 1) translation of the AIR scale into Chinese; 2) validity of the AIR scale section; and 3) reliability of the scale.

\section{Methods \\ Participants}

A two-center study was conducted in two tertiary hospitals in Tianjin, China. From October 2015 to April 2016, 202 patients with COPD who attended the outpatient service were asked to participate in the study. A convenience sample of 181 patients with COPD agreed to participate and were recruited for the study. Participants were eligible if they met the following criteria: 1) 40 years of age or older; 2) diagnosis of COPD (according to the standards set forth by the GOLD); ${ }^{1} 3$ ) willing to participate and sign written informed consent; and 4) ability to understand the items in the scales and write mandarin. The exclusion criteria were as follows: 1) documented psychological disorders (according to the diagnosis of the Mini-International Neuropsychiatric Interview); 2) had no ability to communicate normally; and 3) had another severe disease (severe pulmonary disease and/or cardiac disease). Of the 181 patients, 31 patients, who visited hospital regularly, were randomly selected to complete a second test for calculating the test-retest reliability with the AIR-C (AIR-Chinese version) scale 2 weeks later.

\section{Procedure}

The procedures in this study were approved by the research ethics committees of Tianjin Medical University. All participants were informed about the purpose of the study and also signed the informed consent.

\section{Translation of the AIR-C scale}

With the approval of the original authors, according to the guidelines described by Beaton et al, ${ }^{21}$ the English version of the AIR scale was translated into Chinese. First, the original AIR scale was translated into Chinese by two experts who were proficient in English and Chinese and 
specialized in COPD (T1) and psychiatry (T2), and then the two Chinese drafts (T1 and T2) were reviewed by a third medical professional to formulate a compromised forward translated version (AIR-C scale). Then, the AIR-C scale underwent a backward translation into English by another two independent professionals (one an English speaker and the other who lived in an English-speaking country for $>10$ years). The reverse translation was performed to ensure no conceptual discrepancies existed, and the same procedure was repeated until all the keywords were suitable. Finally, the AIR-C scale was evaluated by six patients with COPD and two doctors who reviewed and edited the sentences in the scale to maintain the appropriate meaning for both cultures. We established the final Chinese version of the AIR scale.

All participants were asked to complete a battery of five self-report measures: demographic variables, the AIR-C scale and the HADS to measure anxiety status, the Clinical COPD Questionnaire (CCQ) to measure quality of life, and the Activities of Daily Living Scale (ADLS) to measure function limitations. All participants completed these instruments at the time of enrollment; disease characteristics (age, gender, educational level, smoking status, the frequency of exacerbations in the last year, and so on) were assessed in the same period. Assessment was conducted in a quiet room of the outpatient service of the hospital, and the time taken was less than 20 minutes. To test the testretest reliability, of the 181 patients, 31 patients, who visited hospital regularly, were randomly selected to complete a second test for calculating the test-retest reliability with the AIR-C scale 2 weeks later. Measures are described in the following section.

\section{Measures}

\section{Demographic characteristics}

The demographic information sheet developed for this study included sociodemographic items and clinical history, including age, gender, educational level, comorbidity, smoking status, and the frequency of exacerbations in the last year.

\section{Anxiety Inventory for Respiratory Disease}

The AIR scale has been designed using both emic and etic perspectives, and it incorporates the words of patients. It contains 10 items with 4-point response options from 0 (no anxiety symptom at all) to 3 (almost all of the time). The score range is from 0 to 30 , and the high score responds to elevated symptoms of anxiety in patients with COPD. It is a valid and reliable scale and has good psychometric properties with a cutoff score of $\geq 8$ on the AIR showing a sensitivity of 0.8 , a specificity of 0.75 , a positive predictive value of $67 \%$, and a negative predictive value of $81 \%{ }^{18,19}$ A new study, which examined the responsiveness of the AIR scale in PR, conducted by Professor Yohannes and his team, indicated that the AIR scale is sensitive to change following PR in patients with COPD and can be used in future studies evaluating interventions that reduce anxiety in COPD. ${ }^{20}$

\section{The Hospital Anxiety and Depression Scale}

The HADS was used to measure anxiety and depression. It consists of 14 items grouped into two subscales to measure anxiety (HADS-A) and depression (HADS-D), respectively. ${ }^{22}$ Items are scored on a four-step scale ranging from 0 to 3 ( 0 means not at all and 3 means very much). The total score ranges from 0 to 21 . Based on previous studies, higher scores indicated elevated anxiety and depression levels. A total score ranging from 0 to 7 represents normal symptom levels, a total score ranging from 8 to 10 indicates probable anxiety or depression symptoms, and a total score $\geq 11$ indicates clinically elevated anxiety or depression. ${ }^{23,24}$ The HADS is widely used to assess anxiety and depression, with good validity and reliability (Cronbach's $\alpha=0.78$ for HADS-A and Cronbach's $\alpha=0.79$ for HADS-D) for use in clinical situations..$^{24,25}$ The Chinese version of HADS has been developed and validated by a previous study. ${ }^{26}$

\section{The Clinical COPD Questionnaire}

The health-related quality of life was measured by the CCQ. The CCQ is self-administered, user-friendly, and contains only 10 items, subdivided into three domains: symptoms state (items 1, 2, 5, and 6), functional state (items 7, 8, 9, and 10), and mental state (items 3 and 4). Participants responded to each question using a 7-point scale from $0=$ asymptomatic or no-limitation to $6=$ extremely symptomatic or totally limited. ${ }^{27}$ Questions are scored on a scale of 0 to 6 , with a higher score indicating worse health status. The original research indicated the CCQ was a reliable, valid tool for measuring health-related quality of life in patients with COPD ${ }^{27}$ The CCQ was translated into more than 60 languages worldwide and also was validated to show it had good psychometric properties. ${ }^{28,29}$

\section{The Activities of Daily Living Scale}

The ADLS was used to measure the patient's functional ability, which consists of 14 items across two domains: Physical 
Self-Maintenance Scale (PSMS) and Instrumental Activities of Daily Living Scale (IADL). Patients are asked to indicate their ability to perform activities across four possible responses: not at all, with help, alone with difficulty, or alone easily. Scores range from 16 to 64, with a higher score indicating worse functional ability. The cutoff score of the ADLS is $14 .^{30}$

\section{Statistical analysis}

All data analysis was performed using SPSS, version 19.0 (IBM Corporation, Armonk, NY, USA). Continuous variables were described as mean (SD) or median (first and third quartiles Q1-Q3). Categorical variables were expressed as relative frequencies and proportions. The level of statistical significance was set at $P<0.05$.

\section{Reliability}

Reliability was estimated by two aspects: one was internal consistency through the value of Cronbach's $\alpha$ for the total score of the AIR-C scale and the other was test-retest reliability, which was done by calculating the intra-class correlation coefficient (ICC) for absolute agreement between two tests. ${ }^{31,32}$ A Cronbach's $\alpha$ of $>0.70$ was recognized acceptable internal consistency, $>0.8$ indicated good, and $>0.9$ meant excellent; ${ }^{33,34}$ and an ICC score $>0.75$ indicated excellent reliability. ${ }^{35}$

\section{Validity}

Validity is a quantitative assessment of how well the scale measures what it claims to measure. ${ }^{36}$ The validity of the AIR-C scale was based on content validity, convergent validity, and construct validity. The Content Validity Index (CVI) was supported by six experts, including two nurses and four doctors. Four doctors were specialized in COPD, psychology, and psychiatric field. Two nurses were specialized in COPD and were familiar with the subject of anxiety. We also invited three COPD patients to evaluate the CVI of the scale. All experts had at least 10 years of working experience in a tertiary hospital. A four-point expert rating scale was used, where 4 represented very relevant, 3 represented strong relevance, 2 represented weak relevance, and 1 represented not relevant. Both scale-level CVI/universal agreement (S-CVI/UA) and scale-level CVI/average agreement (S-CVI/Ave) were calculated. S-CVI/UA was calculated as the proportion of the number of ratings of three or four items by the total number of the scale; S-CVI/Ave was tested by taking the average of the item-level CVIs (I-CVIs). ${ }^{37}$ Several previous studies indicated that a scale with an I-CVI value $\geq 0.78$ was recognized acceptable, $\mathrm{S}-\mathrm{CVI} / \mathrm{UA}>0.8$ means good, and an S-CVI/Ave was expected to achieve $0.90 .38,39$

Convergent validity was investigated by calculating the correlation coefficients of the AIR-C scale with the HADS, ADLS, and CCQ. Spearman's correlation coefficients were used, where $r<0.30$ meant low, $0.30<r<0.60$ meant moderate, and $r>0.60$ meant high. ${ }^{33}$

Confirmatory Factor Analysis (CFA) was performed to assess the construct validity, which was performed with Analyse of Moment Structures (version19) and was conducted to assess the goodness of fit of the model to the data. Six important indices were used to evaluate the fit of the models. According to previous studies, the fit of the models was considered acceptable if the Comparative Fit Index (CFI) was $\geq 0.90$, root mean square error of approximation (RMSEA) was between 0.08 and 0.10 , Incremental Fit Index (IFI) was $>0.90$, and the Tucker-Lewis Index (TLI) was $\geq 0.80 .{ }^{40-42} \mathrm{~A}$ better fit was suggested if the Goodness-of-Fit Index (GFI) was $\geq 0.90$, CFI and TLI were $\geq 0.95$, RMSEA was $<0.08$, and root mean square residual $(\mathrm{RMR})$ was $<0.05$. $^{43}$

\section{Measurement error}

Measurement error is the random and systematic error of a subject's situation that does not reflect true change in the construct to be measured. To distinguish the measured change with the true change, standard error of measurement (SEM) is too important to be ignored. The SEM was calculated based on the sample SD and the calculated ICC according to the following formula: ${ }^{44}$

$$
\mathrm{SEM}=\mathrm{SD} \sqrt{1-\mathrm{ICC}}
$$

\section{Results}

\section{Participants' characteristics}

A total of 181 patients with moderate-to-severe COPD were recruited for the study between October 2015 and April 2016. Participants' characteristics are summarized in Table 1. The mean (SD) age of the participants was 67.21 (8.1) years, and $67.4 \%$ were males. Patients' scores on the AIR-C scale, the ADLS, the HADS, and the CCQ are also listed in Table 1. There were no patients withdrawn in the follow-up 2-week period, because the patients had regular visiting to the outpatient clinic for taking medicine and were willing to continue participation in the present study.

\section{Reliability}

The internal consistency (Cronbach's $\alpha$ ) of the AIR-C scale for the subscales and the total score was 0.91 (95\% CI, $0.89-$ 0.93). Test-retest reliability of the AIR-C scale was calculated 
Table I COPD patients' characteristics $(\mathrm{N}=\mid 81)$

\begin{tabular}{|c|c|c|}
\hline Characteristics & $\mathbf{n}$ & $\% /$ mean (SD) \\
\hline Age (years) & 181 & $67.21(8.1)$ \\
\hline \multicolumn{3}{|l|}{ Gender } \\
\hline Female & 59 & 32.6 \\
\hline Male & 122 & 67.4 \\
\hline Body mass index $\left(\mathrm{kg} / \mathrm{m}^{2}\right)$ & 181 & $23.89(4.08)$ \\
\hline \multicolumn{3}{|l|}{ Comorbidities } \\
\hline None & 57 & 31.5 \\
\hline I & 69 & 38.1 \\
\hline$\geq 2$ & 55 & 30.4 \\
\hline \multicolumn{3}{|l|}{ Smoking status } \\
\hline Never & 44 & 24.3 \\
\hline Ex-smoker & 114 & 63.0 \\
\hline Current smoker & 23 & 12.7 \\
\hline \multicolumn{3}{|l|}{ MRC dyspnea scale } \\
\hline 0 & 14 & 7.7 \\
\hline I & 22 & 12.2 \\
\hline 2 & 72 & 39.8 \\
\hline 3 & 50 & 27.6 \\
\hline 4 & 23 & 12.7 \\
\hline \multicolumn{3}{|c|}{ Frequency of exacerbations in the last year } \\
\hline 0 & 39 & 21.5 \\
\hline I & 70 & 38.7 \\
\hline 2 & 43 & 23.8 \\
\hline$\geq 3$ & 29 & 16 \\
\hline Grades of COPD & 150 & \\
\hline GOLD I & 4 & 2.67 \\
\hline GOLD 2 & 52 & 34.67 \\
\hline GOLD 3 & 64 & 42.68 \\
\hline GOLD 4 & 30 & 19.98 \\
\hline Total score of AIR & 181 & $7.15(3-11)$ \\
\hline Total score of CCQ & 181 & $2.53(0.86)$ \\
\hline Total score of HADS & 181 & $12.56(7.89)$ \\
\hline HADS-A & & $4.83(3.88)$ \\
\hline HADS-D & & $7.73(4.62)$ \\
\hline Total score of ADLS & 181 & $21.65(14.5-25)$ \\
\hline Somatic dimension & & $7.7(6-8)$ \\
\hline Instrument dimension & & $13.95(8.5-17)$ \\
\hline
\end{tabular}

Notes: Continuous variables are expressed as mean (standard deviation) or median (first and third quartiles QI-Q3), and categorical variables are expressed as relative frequencies and proportion.

Abbreviations: MRC, Medical Research Council; GOLD, Global Initiative for Chronic Obstructive Lung Disease; AIR, Anxiety Inventory for Respiratory Disease; CCQ, Clinical COPD Questionnaire; HADS, Hospital Anxiety and Depression Scale; A, anxiety; D, depression; ADLS, Activities of Daily Living Scale.

from the responses of the 31 patients who reported no major change in their health and mental status after 2 weeks; the intraclass correlation coefficient was $0.90(P<0.001)$. The SEM for the total score of the AIR-C scale was 1.6.

\section{Validity}

The content validity rate was in the range of $0.89-1$; the values of the S-CVI/UA and S-CVI/Ave were 0.90 and 0.98 , respectively. The three patients judged that the content in the scale were appropriate and comprehensive. Table 2 reveals experts' ratings and CVI calculation.
Table 2 Experts' ratings and CVI calculation $(n=9)$

\begin{tabular}{|c|c|c|c|c|c|c|c|c|c|c|}
\hline \multirow[t]{2}{*}{ Item } & \multicolumn{9}{|c|}{ Experts' ratings ${ }^{a}$} & \multirow[t]{2}{*}{$\mathrm{I}-\mathrm{CV} \mathrm{I}^{\mathrm{b}}$} \\
\hline & $\mathbf{A}$ & B & C & D & $\mathbf{E}$ & $\mathbf{F}$ & G & $\mathbf{H}$ & $\mathbf{I}$ & \\
\hline $\mathrm{HI}$ & 4 & 3 & 3 & 4 & 3 & 4 & 4 & 3 & 3 & I \\
\hline $\mathrm{H} 2$ & 4 & 4 & 4 & 4 & 4 & 4 & 4 & 4 & 4 & I \\
\hline $\mathrm{H} 3$ & 4 & 4 & 4 & 4 & 4 & 4 & 4 & 4 & 4 & I \\
\hline $\mathrm{H} 4$ & 4 & 4 & 4 & 4 & 4 & 4 & 3 & 3 & 4 & 1 \\
\hline H5 & 4 & 4 & 3 & 2 & 3 & 4 & 3 & 3 & 4 & 0.89 \\
\hline $\mathrm{H} 6$ & 3 & 4 & 4 & 4 & 4 & 4 & 4 & 4 & 4 & 1 \\
\hline $\mathrm{H} 7$ & 4 & 4 & 4 & 4 & 4 & 4 & 4 & 4 & 4 & I \\
\hline H8 & 4 & 4 & 4 & 4 & 4 & 4 & 4 & 4 & 4 & I \\
\hline $\mathrm{H} 9$ & 4 & 4 & 4 & 4 & 4 & 4 & 4 & 4 & 4 & I \\
\hline $\mathrm{HIO}$ & 3 & 4 & 4 & 4 & 4 & 4 & 4 & 4 & 4 & 1 \\
\hline
\end{tabular}

Notes: aNine expert ratings for the I0-item AIR scale. ${ }^{b}$ According to the criteria for $\mathrm{I}-\mathrm{CVI}$, a scale with an I-CVI value $\geq 0.78$ was considered acceptable.

Abbreviations: CVI, content validity index; I-CVI, item-level CVI; AIR, Anxiety Inventory for Respiratory Disease.

The convergent validity was examined by calculating the Spearman's correlation coefficient between the AIR-C scale and the score of the HADS, the CCQ, and the ADLS. The AIR-C scale scores showed high correlations with HADSTotal $(r=0.83, P<0.01)$, HADS-A $(r=0.81, P<0.01)$, and HADS-D $(r=0.76, P<0.01)$. In addition, the correlation coefficients with the CCQ and the ADLS were $0.44(P<0.01)$ and $0.36(P<0.01)$, respectively. Table 3 shows the correlations between the total scores of the AIR-C scale and other self-reported scales.

In addition, the construct validity was examined by means of CFA. Two two-factor models were conducted (Figures 1 and 2), and the two-factor model (Figure 2B, model 3) showed a better model fit, with TLI, IFI, and CFI reaching acceptable fit $(0.95,0.97,0.97)$. The analysis of the scale produced GFI value of 0.93 . Model adjustment according to modification indices suggested two minor adjustments to the two-factor model: item 10 only belonging to the general anxiety (Figure 2A, model 2) and allowing error terms in items 3 and 7, 6 and 10, and 3 and 10 to covary (Figure 2B, model 3). Both modifications fit within the conceptualized model. Model 3 demonstrated the best fit, with six indicators

Table 3 Spearman's $\rho$ correlations of AIR with other measures

\begin{tabular}{ll}
\hline Measure & AlR total \\
\hline HADS-Total & 0.83 \\
HADS-A & $0.8 I^{*}$ \\
HADS-D & 0.76 \\
ADLS & $0.36^{*}$ \\
CCQ & $0.44^{*}$ \\
\hline
\end{tabular}

Note: *Correlation is significant at the 0.05 level.

Abbreviations: AIR, Anxiety Inventory for Respiratory Disease; HADS, Hospital Anxiety and Depression Scale; HADS-A, Hospital Anxiety and Depression ScaleAnxiety; HADS-D, Hospital Anxiety and Depression Scale-Depression; ADLS, Activities of Daily Living Scale; CCQ, Clinical COPD Questionnaire. 


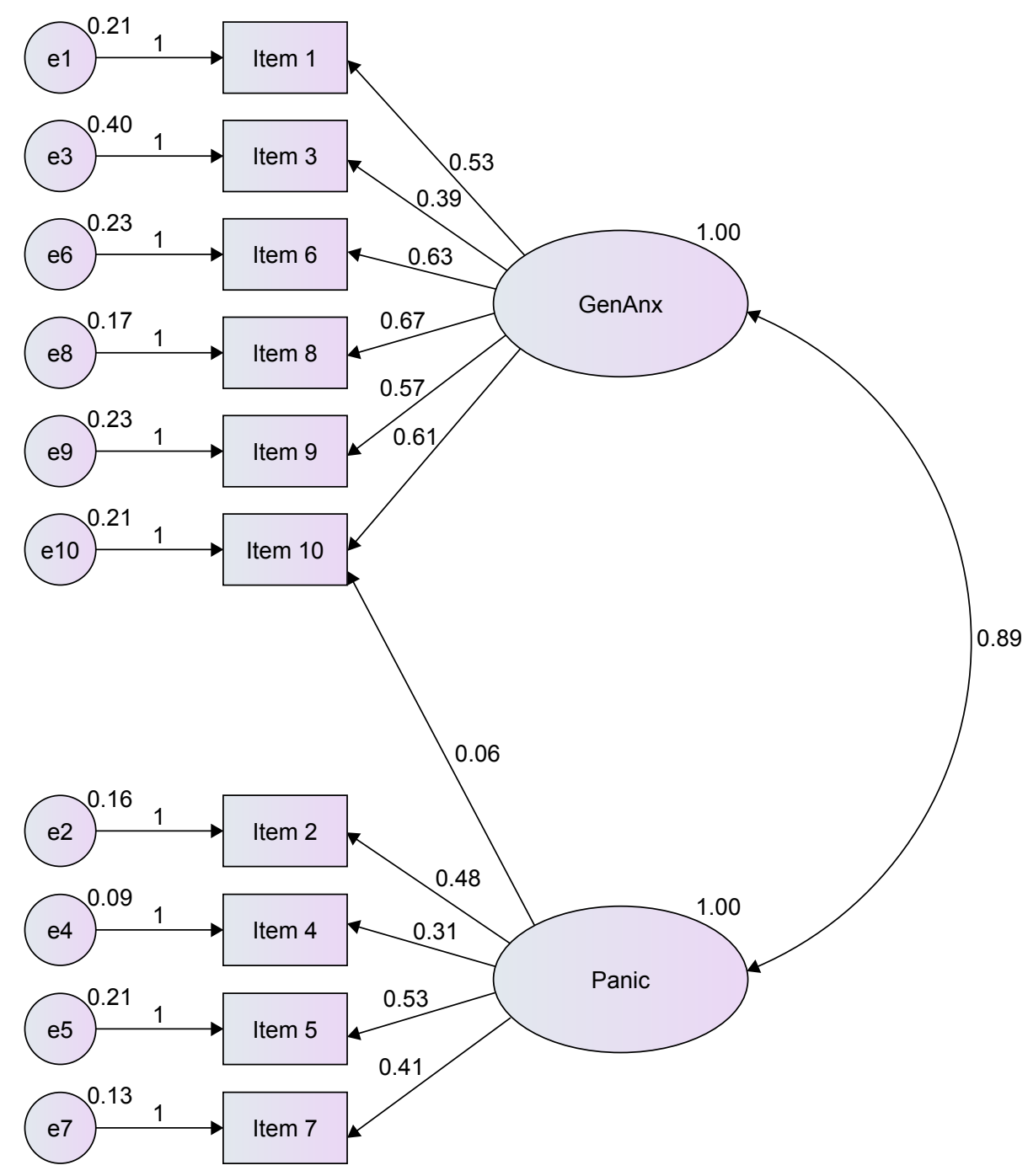

Figure I Model I, two-factor model.

Note: Item 10 belongs to both the Panic and the GenAnx.

Abbreviation: GenAnx, general anxiety.

suggesting good fit to the data. Table 4 shows the CFA index of the AIR-C scale.

\section{Discussion}

The purpose of our study was to translate the English version of the AIR scale into the Chinese version and to examine the psychometric properties of the AIR-C scale in a sample of patients with COPD in China. To our knowledge, this is the first time the psychometric properties of the AIR-C scale have been examined in a Chinese COPD population. The results of the current study indicate that the AIR-C scale has appropriate psychometric properties and is a relatively reliable and valid scale for measuring the anxiety in patients with COPD in China.

The results from the current study supported both the reliability and validity of the AIR-C scale. The intra-class correlation coefficient of the AIR-C scale was 0.90 in this study, reflecting its excellent test-retest reliability, ${ }^{35}$ and the internal consistency of the scale was acceptable (with Cronbach's $\alpha$ coefficient of 0.91). ${ }^{34}$ Our assessment of internal consistency and test-retest reliability revealed that the AIR-C scale is as reliable as the original version (Cronbach's $\alpha$ coefficient $=0.92$ and intra-class correlation coefficient $=0.81) .{ }^{18}$

The AIR-C scale also showed good content validity in this study. The I-CVI of 0.78 was the cutoff for either removing or retaining an item, established in a study with nine experts. ${ }^{38,39}$ In the present study, the I-CVI of all items except H5 was 1 (the I-CVI of H5 was 0.89); according to the criteria of the content validity, the H5 was retained. Meanwhile, the convergent validity of the AIR-C scale was supported by significant correlations between the AIR-C scale and the other 
self-reported scales, including CCQ, HADS, and ADLS. The correlation coefficients between AIR-C scale scores and CCQ, HADS, and ADLS scores showed significant and moderate-to-high correlations $(0.36-0.83)$ in the present study, indicating good convergent validity. ${ }^{33}$ Compared with the original research, the correlation between AIR-C scale and ADLS was low ( $r=0.36$; original research, $r=0.52$ ). One possible reason for this finding is that the ADLS is not the specific instrument for measuring the functional status in patients with COPD, and there is no disease-specific ADLS in China. ${ }^{45}$

In terms of construct validity, CFA demonstrated that a two-factor model had the best fit. According to the original study using this scale, the two-factor model had the best fit, with TLI reaching acceptable fit and other indicators suggesting good fit to the data. Besides, the factor structure was consistent with the original authors' conceptual model, which included 10 items that reflected symptoms of both panic (including items 2, 4, 5, 7, and 10) and general anxiety (including items $1,3,6,8,9$, and 10). ${ }^{18}$ In this study, as the scale was specifically designed to include items that reflect symptoms of both panic and general anxiety, the two types of two-factor model were examined. Compared with the original research, ${ }^{18}$ our findings indicated that when the 10 items belong to the general anxiety (the original research indicated that the 10 items belong to both the general anxiety and the panic), the modified two-factor model showed better model fit. The possible reason was that the sample size of the original study was relatively small to conduct a CFA; ${ }^{42}$ however, there were enough participants for conducting the CFA in the present study.

A recent meta-analysis by Coventry and $\mathrm{Hind}^{46}$ suggested that a PR program that incorporates exercise, education, and social support significantly reduced anxiety compared with

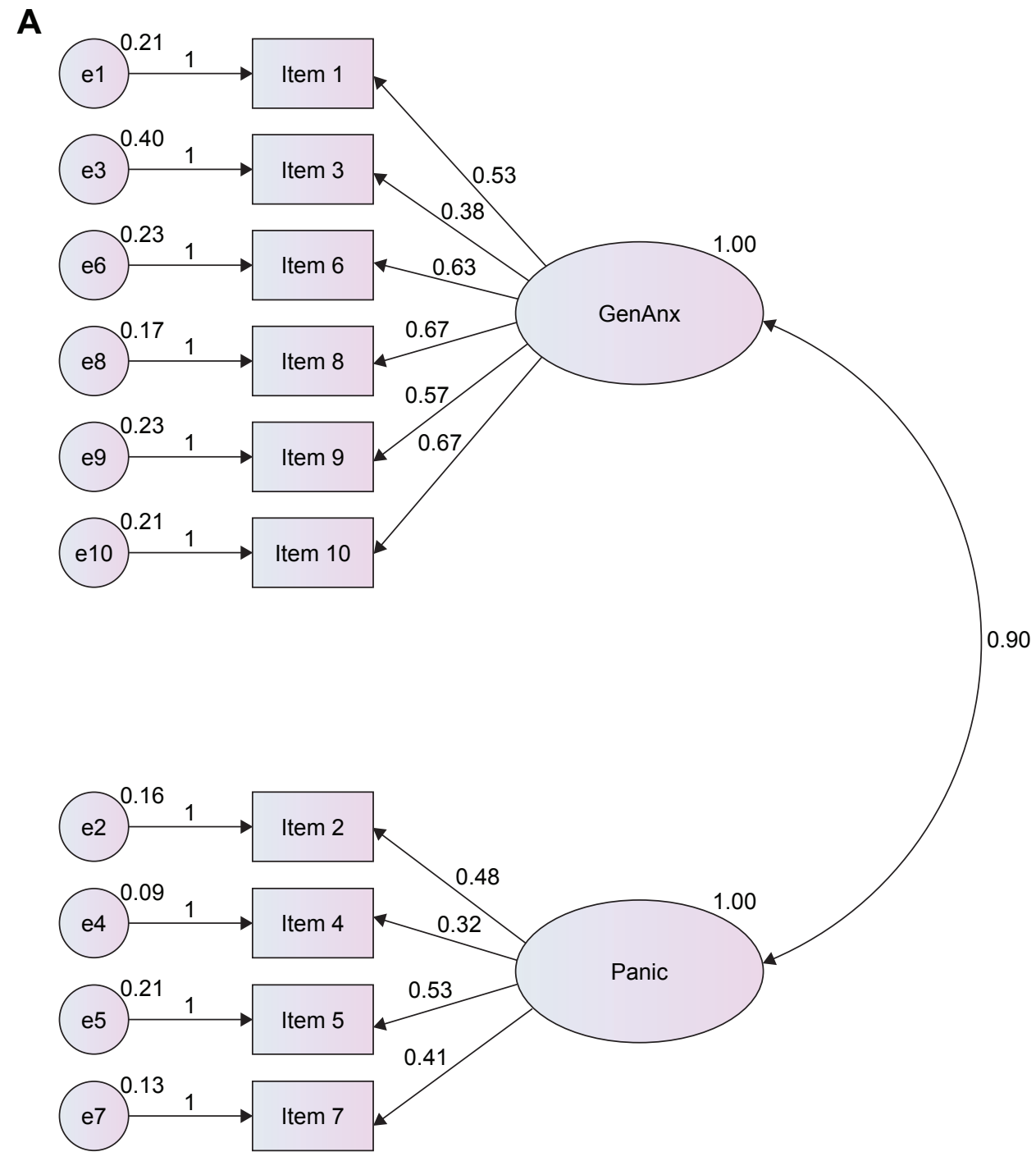

Figure 2 (Continued) 


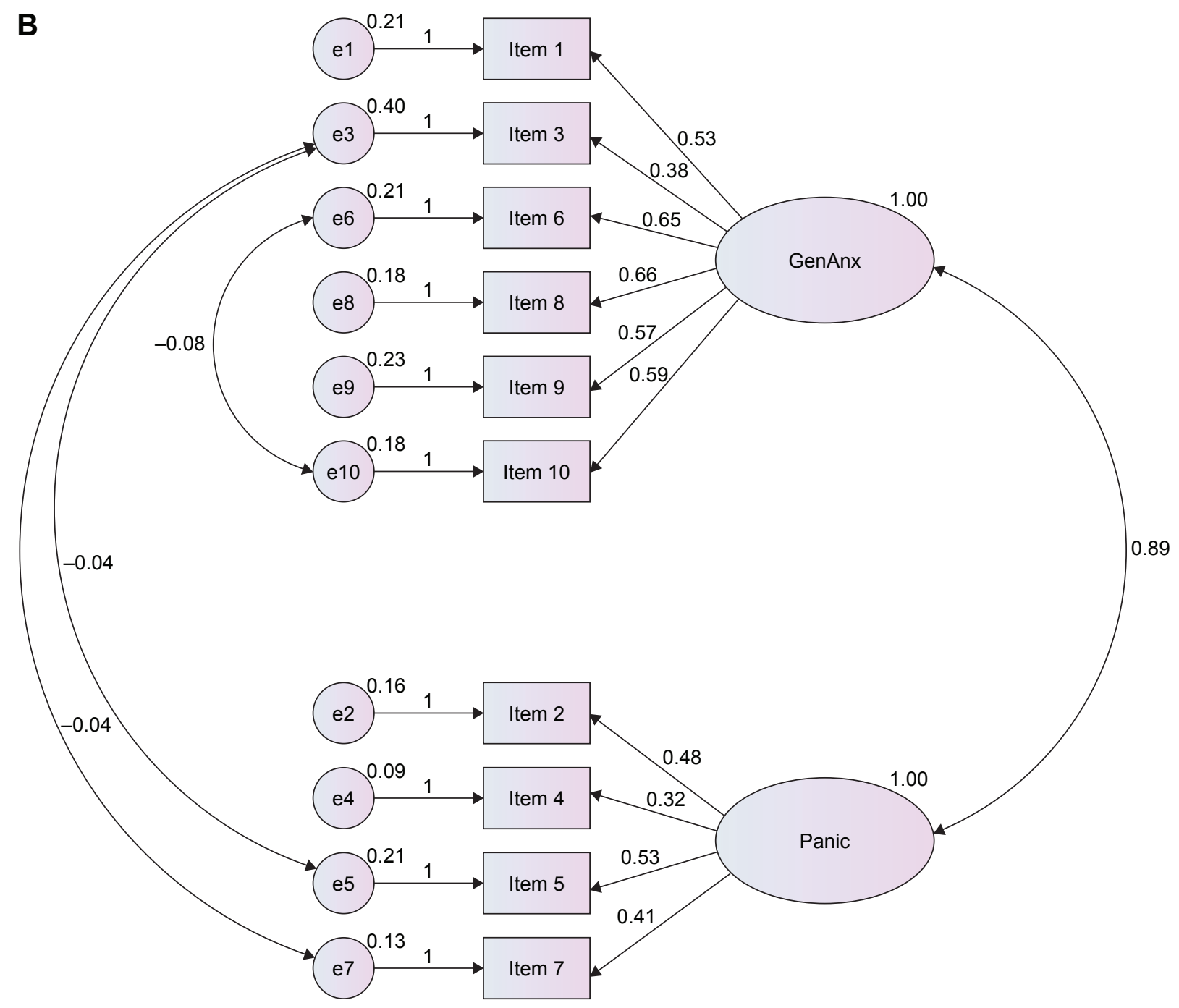

Figure 2 Model 2 and Model 3, modified two-factor models for AIR-C.

Notes: (A) Model 2, two-factor model. Item 10 belongs to the GenAnx. (B) Model 3, modified two-factor models (model 2) for AIR, allowing error terms in items 3 and 7,6 and 10 , and 3 and 10 to covary.

Abbreviations: AIR-C, Anxiety Inventory for Respiratory Disease-Chinese version; GenAnx, generalized anxiety.

standard care in patients with COPD, but it is not clear which aspects of the PR program have the impact upon anxiety symptoms. A new study, conducted by Professor Yohannes and his team, which observed the responsiveness of the AIR scale following 8-week PR, indicated that the AIR scale was responsive to the effects of $\mathrm{PR}$ and that the mean change in

Table 4 Model fit indices for the four models of the AIR-C scale

\begin{tabular}{lllllll}
\hline Model & TLI & GFI & IFI & CFI & RMSEA & RMR \\
\hline Model I & 0.92 & 0.90 & 0.94 & 0.94 & 0.1 & 0.02 \\
Model 2 & 0.92 & 0.90 & 0.94 & 0.94 & 0.09 & 0.02 \\
Model 3 & 0.95 & 0.93 & 0.97 & 0.97 & 0.07 & 0.01 \\
\hline
\end{tabular}

Abbreviations: TLI, Tucker-Lewis Index; GFI, Goodness-of-Fit Index; IFI, Incremental Fit Index; CFI, Comparative Fit Index; RMSEA, root mean square error of approximation; RMR, root mean square residual; AIR-C, Anxiety Inventory for Respiratory Disease-Chinese version. the AIR score was significantly associated with changes in dyspnea and quality-of-life scores. ${ }^{20}$ In the current study, although we did not include a validated measure of anxiety, the scores on the AIR-C scale indicate that patients with COPD experience a range of anxiety symptoms in China. Further studies are needed to examine the responsiveness of the AIR-C scale to PR programs.

Several limitations need to be addressed in this study. First, this was a cross-sectional study, and longitudinal research will contribute to validate the sensitivity of anxiety to clinical variables in the future. Second, the participants were considered to be representative of Chinese patients with COPD who live in Tianjin city and they may not be generalized to the overall population in China; a future study should recruit more participants from different regions in China 
to replicate these findings. Third, the main gender of the participants who were recruited in the study was male. The results cannot be generalized to total patients with COPD in China, therefore, future studies should include female patients with COPD. Fourth, the Mini-International Neuropsychiatric Interview was not used as a "gold standard" diagnostic tool in this study. In this regard, a future study could provide a gold standard diagnosis in order to improve the accuracy for the clinical anxiety in patients with COPD.

\section{Conclusion}

The Chinese version of the AIR (AIR-C) has shown acceptable levels of psychometric properties, and it is a reliable and valid instrument for measuring anxiety in Chinese COPD patients. As a disease-specific non-somatic anxiety measurement, it might be useful as a sensitive measure for measuring anxiety in Chinese COPD patients.

\section{Acknowledgments}

The authors are grateful for the financial support from the Humanities and Social Science Research of China (Grant No 15YJA2H109). The authors would like to express their gratitude to all the subjects for their kind participation in the study.

\section{Author contributions}

All listed authors contributed toward data analysis, drafting, and revising the paper and agree to be accountable for all aspects of the work.

\section{Disclosure}

The authors report no conflicts of interest in this work.

\section{References}

1. Global Initiative for Chronic Obstructive Lung Disease [homepage on the Internet]. Global Strategy for the Diagnosis, Management, and Prevention of Chronic Obstructive Pulmonary Disease. 2016. Available from: http://www.goldcopd.com. Accessed January 31, 2016.

2. World Health Organization. Chronic respiratory diseases. Available from: http://www.who.int/respiratory/copd/burden/en/. Accessed November 4, 2013.

3. Zhong N, Wang C, Yao W, et al. Prevalence of chronic obstructive pulmonary disease in China: a large, population-based survey. Am J Respir Crit Care Med. 2007;176(11):753-760.

4. Dahlen I, Janson C. Anxiety and depression are related to the outcome of emergence treatment in patients with obstructive pulmonary disease. Chest. 2002;122(5):1633-1637.

5. Eisner MD, Blanc PD, Yelin EH, et al. Influence of anxiety on health outcomes in COPD. Thorax. 2010;65(3):229-234.

6. Felker B, Bush KR, Harel O, et al. Added burden of mental disorders on health status among patients with chronic obstructive pulmonary disease. Prim Care Companion J Clin Psychiatry. 2010;12(4):pii:PCC.09m00858.

7. Yohannes A, Baldwin R, Connolly M. Mood disorder in elderly patients with chronic obstructive pulmonary disease. Rev Clin Gerentol. 2000;10(2): 193-202.
8. Willgoss TG, Yohannes AM. Anxiety disorders in patients with COPD: a system review. Respir Care. 2013;58(5):858-866.

9. Panagioti M, Scott C, Blakemore A, Coventry PA. Overview of the prevalence, impact, and management of depression and anxiety in chronic obstructive pulmonary disease. Int J Chron Obstruct Pulmon Dis. 2014; 9:1289-1360.

10. Zhang L, Lou P, Chen PP, et al. Impact of risk factors, activities and psychological disorders on the health of patients with chronic obstructive pulmonary disease in China: a cross-sectional study. BMC Public Health. 2013;13:627.

11. Roundy K, Cully JA, Stanley MA, et al. Are anxiety and depression addressed in primary care patients with chronic obstructive pulmonary disease? A chart view. Prim Care Companion J Clin Psychiatry. 2005; 7(5):213-218.

12. Yohannes AM, Willgoss T, Baldwin RC, et al. Depression and anxiety in chronic heart failure and chronic obstructive pulmonary disease: prevalence, relevance and management principles. Int $J$ Geriatr Psychiatry. 2010;25(12):1209-1221.

13. Kunik ME, Roundy K, Veazey C, et al. Surprisingly high prevalence of anxiety and depression in chronic breathing disorders. Chest. 2005; 127(4):1205-1211.

14. Li YJ, Yuan T, Xu MF. [Clinical analysis of elderly chronic patients with depression and anxiety disorders.] Chin J Gerontol. 2006;26(9): 1263-1264. [in Chinese].

15. Ji X, Qian GS. [Emphasis on diagnosis and treatment of anxiety and depression in patients with COPD.] Chin J Lung Dis. 2011;4(3):170-172. [in Chinese]

16. Martin CR. What does the hospital anxiety and depression scale (HADS) really measure in liaison psychiatry setting? Curr Psychiatr Rev. 2005;1(1):69-73.

17. Steer RA, Willman M, Kay PAJ, et al. Differentiating elderly medical and psychiatric outpatients with the Beck Anxiety Inventory. Assessment. 1994;1(4):345-351.

18. Willgoss TG, Goldbart J, Fatoye F, Yohannes AM. The development and validation of the anxiety inventory for respiratory disease. Chest. 2013;5(144):1587-1596

19. Yohannes AM, Willgoss TG. The accuracy of the anxiety inventory for respiratory disease scale for patients with COPD. Int $J$ Geriatr Psychiatry. 2015;30(1):106-108.

20. Yohannes AM, Dryden S, Hanania NA. The responsiveness of the anxiety inventory for respiratory disease to pulmonary rehabilitation. Chest. 2016;150(1):188-195.

21. Beaton DE, Bombardier C, Guillemin F. Guidelines for the process of cross-cultural adaptation of self report measures. Spine (Phila Pa 1976). 2000;25(24):3186-3191

22. Zigmond AS, Snaith RP. The hospital anxiety and depression scale. Acta Psychiatr Scand. 1983;67(6):361-370.

23. Olssøn I, Mykletun A, Dahl AA. The hospital anxiety and depression rating scale: a cross-sectional study of psychometrics and case finding abilities in general practice. BMC Psychiatry. 2005;5:46.

24. Mykletun A, Stordal E, Dahl AA. Hospital anxiety and depression (HAD) scale: factor structure, item analyses and internal consistency in a large population. Br J Psychiatry. 2001;179:540-544.

25. Johnston M, Pollard B, Hennessey P. Construct validation of hospital anxiety and depression scale with clinical populations.J Psychosom Res. 2000;48(6):579-584.

26. Zheng LL, Wang YL, Li HL. Application of hospital anxiety and depression scale in general hospital: an analysis in reliability and validity. Shanghai Archiv Psychiatr. 2003;15:264-266.

27. van der Molen T, Willemse BW, Schokker S, ten Hacken NH, Postma DS, Juniper EF. Development, validity and responsiveness of the clinical COPD questionnaire. Health Qual Life Outcomes. 2003;1:13.

28. Reda AA, Kotz D, Kocks JW, Wesseling G, van Schayck CP. Reliability and validity of the clinical COPD questionnaire and chronic respiratory questionnaire. Respir Med. 2010;104(11):1675-1682.

29. Damato S, Bonatti C, Frigo V, et al. Validation of the clinical COPD questionnaire in Italian language. Health Qual Life Outcomes. 2005;3:9. 
30. Lawton MP, Brody EM. Assessment of older people: self-maintaining and instrumental activities of daily living. Gerontologist. 1969;9(3 pt 1): 179-186.

31. Yu HM, Luo YH, Sa J, et al. Intraclass correlation coefficient and software procedures. Chin J Health Stat. 2011;28:497-500.

32. Shrout PE, Fleiss JL. Intraclass correlations: uses in assessing rater reliability. Psychol Bull. 1979;86(2):420-428.

33. Streiner DL, Norman RG. Health Measurement Scales: A Practical Guide to Their Development and Use. 3rd ed. Oxford: Oxford University Press; 2003.

34. Fayers PM, Machi D. Quality of Life: Assessment, Analysis and Interpretation. New York: John Wiley \& Sons, Inc; 2000.

35. Marx RG, Menezes A, Horovitz L, Jones EC, Warren RF. A comparison of two time intervals for test-retest reliability of health status instruments. J Clin Epidemiol. 2003;56(8):730-735.

36. De Boer A, Wijker W, Speelaman J, De Haes J. Quality of life in patients with Parkinson's disease: development of a questionnaire. J Neurol Neurosurg Psychiatry. 1996;61(1):70-74.

37. Polit DF, Beck CT, Owen SV. Is the CVI an acceptable indicator of content validity? Appraisal and recommendations. Res Nurs Health. 2007;30(4):459-467.

38. Lynn MR. Determination and quantification of content validity. Nurs Res. 1986;35(6):382-385.
39. Waltz CF, Strickland OL, Lenz ER. Measurement in Nursing and Health Research. 3rd ed. New York: Springer; 2005:157.

40. Hooper D, Couglan J, Mullen MR. Structural equation modelling: guidelines for determining model fit. Electron J Bus Res Methods. 2008; 6(1):53-60.

41. Hu LT, Bentler PM. Cut off criteria for fit indexes in covariance structure analysis: conventional criteria versus new alternatives. Struct Equ Modeling. 1999;6(1):1-55.

42. Kline RB. Principles and Practice of Structural Equation Modelling. 2nd ed. New York, NY: The Guildford Press; 2005.

43. Miles J, Shevlin M. Effects of sample size, model specification and factor loadings on the GFI in confirmatory factor analysis. Pers Individ Dif. 1998;25(1):85-90.

44. Atkinson G, Nevill AM. Statistical methods for assessing measurement error (reliability) in variables relevant to sports medicine. Sports Med. 1998;26(4):217-238.

45. Chen SY, Meng S. Assessment of activities of daily living for patients with chronic obstructive pulmonary disease [in Chinese]. Chin J Rehabil Theory Pract. 2009;15(5):414-415.

46. Coventry PA, Hind D. Comprehensive pulmonary rehabilitation for anxiety and depression in adults with chronic obstructive pulmonary disease: systematic review and meta-analysis. J Psychosom Res. 2007; 63(5):551-565.
International Journal of COPD

\section{Publish your work in this journal}

The International Journal of COPD is an international, peer-reviewed journal of therapeutics and pharmacology focusing on concise rapid reporting of clinical studies and reviews in COPD. Special focus is given to the pathophysiological processes underlying the disease, intervention programs, patient focused education, and self management protocols.

\section{Dovepress}

This journal is indexed on PubMed Central, MedLine and CAS. The manuscript management system is completely online and includes a very quick and fair peer-review system, which is all easy to use. Visit http://www.dovepress.com/testimonials.php to read real quotes from published authors. 